\title{
Notas DE AQUí Y DE ALLÁ
}

\section{CONGRESOS INTERNACIONALES}

- Universidad de Sao Paolo Brasil, II Congreso Internacional REFMUR "Red de Estudios de Familias Murcia"; del 9 al 15 de septiembre de 2013, María del Pilar Morad de Martínez, presentó la ponencia: Mujeres madres en el vivir trasnacional, entre transgresiones y tradiciones.

- Congreso Internacional de Educación Superior 2014, desarrollado en el Palacio de Convenciones de la Habana, Cuba; del 10 al 14 de febrero de 2014, Las docentes María del Pilar Morad de Martínez, Dora Piñeres De La Ossa y Carolina Hamodi, presentaron las ponencias: 1) Formación para la intervención con familias: Retos Pedagógicos y Sociales. 2) Historia y futuro de la red de evaluación formativa y competitiva en docencia universitaria en España.

- "Encuentro de la Red de Investigadores del Caribe SUE Caribe-UWI Universidad de West Indies, Kingston, Jamaica. Del 10 al 14 de marzo de 2014, las docentes Liris Múnera Cavadía, Dora Piñeres de la Ossa y Ángela Cañón Cárdenas, participaron como investigadoras de los proyectos: "Early childhood education: comparative models of approaches and curricular desingns inthe context of childhood policies", "Women in higher education in the caribean, a comparative perspective: University of Cartagena and University of West indies", "Harressing ecoturism, adaptation strategies to climate change and the creative industries for development: The cases of the Coral National Park and Jamaica" respectivamente.

- Congreso de Trabajo Social, en Manta, Ecuador; el 13 de Marzo de 2014, el docente Alexander Pérez Álvarez, presentó la ponencia: "Retos profesionales para afrontar la cuestión social CELICAI y Universidad Eloy Alfaro de Manabí".

- Encuentro: "Discusiones del movimiento LGBT Cubano/Caribeño y lanzamiento de la red afro-diversidad por el reconocimiento de derechos LGBT en entornos étnico raciales". Desarrollado en La Habana, Cuba; del 02 al 08 de abril de 2014, el docente Alexander Pérez Álvarez, participó como facilitador del encuentro. 
- Murcia, España, I Congreso Internacional de Facultades y Escuelas de Trabajo Social; del 23 al 25 de abril de 2014, la docente Kenia Victoria Cogollo, presento o participo como la ponencia "...".

- "Encuentro de la Red de Soluciones para el Desarrollo Sustentable del Caribe", Kingston, Jamaica. Del 5 al 9 de mayo de 2014, la docente Angela Cañón Cárdenas, participó como investigadora.

- Red Caribbean Sustainable Development Solutions Network (SDSN). University of West Indies (UWI) en Kingston, Jamaica, del 07 y 08 de mayo de 2014, la docente Angela Cañón Cárdenas, presentó la propuesta de proyecto titulada: "Ecoturismo comunitario con base en los sistemas de eco-aldea. Una solución para el desarrollo sostenible del Caribe".

\section{CONGRESOS NACIONALES}

- VI Encuentro Nacional de Formación en Familias año 2013, Universidad del Valle-Cali, del 28 al 30 de agosto de 2013, la docente María del Pilar Morad de Martínez, presentó la ponencia: "La investigación con familias sustento de la acción profesional".

- II Conferencia de Teorías y Literaturas en el Caribe y Latinoamérica, del 9 al 12 de septiembre de 2013, el docente David Lara Ramos, presento la ponencia: "Ceferina Banquez, rupturas en la lírica de los cantos de bullarengue Universidad del Atlántico con el apoyo de la Pontificia Universidad Javeriana".

- VIII Encuentro de Red de Observatorios Regionales de Desarrollo y Paz. Desarrollado en Bogotá, del 18 al 20 de septiembre de 2013, la docente Kenia Victoria Cogollo, participó.

- "Intercambio de experiencias y conversatorio de la mesa de Derechos Humanos del Oriente Antioqueño", del 19 al 20 de noviembre de 2013, la docente Rosa Jiménez Ahumada, participó de la Invitación.

- "Encuentro de intercambio de experiencias investigativas sobre políticas públicas LGBTI". En Pasto, Nariño, del 29 de enero al 01 de febrero de 2014, el docente Alexander Pérez Álvarez, participó como investigador.

- Encuentro de Formadores. Escuela de Reparaciones de la Unidad de Víctimas. En Villeta, Cundinamarca, del 10 al 14 de marzo de 2014, la docente Rosa Jiménez Ahumada, participó de la Invitación. 
- Del 16 al 18 de marzo de 2014, la docente María del Pilar Morad de Martínez, presentó la ponencia: "El cuidado familiar de niños y niñas menores de 12 años: Articulaciones entre grupos familiares, el Estado, el mercado, asl ONG, y las Redes vecinales, según la posición socio-económica en seis ciudades colombianas. Medellín. 